\title{
PSInSAR Improvement using Amplitude Dispersion Index Optimization of Dual Polarimetry Data
}

Mostafa Esmaeili a,* and Mahdi Motagh ${ }^{\text {b }}$

a School of Surveying and Geospatial Engineering, College of Engineering, University of Tehran, Tehran, Iran - maesmaeili@ut.ac.ir ${ }^{\mathrm{b}}$ Department of Geodesy and Remote Sensing, GFZ German Research Center for Geosciences, Potsdam, Germany motagh@gfz-potsdam.de

KEY WORDS: Polarimetric Optimization, Persistent Scatterer Candidate, PSInSAR, Amplitude Dispersion Index (ADI)

\section{ABSTRACT}

Persistent Scatterer Interferometry for SAR data (PSInSAR) improves the ability of conventional InSAR time-series methods by detecting and analysing pixels where the portion of spatiotemporal decorrelations on the phase is negligible. Using dual/quad polarized SAR data provide us with an additional source of information to improve further the capability of InSAR analysis. In this paper, we present a method to enhance PSInSAR using polarimetric optimization method on multi-temporal polarimetric SAR data. The optimization process has been implemented to minimize the Amplitude dispersion Index (ADI) of pixels in SAR images over the time based on the best scattering mechanism. We evaluated the method on a dataset including 17 dual polarization SAR data (HH/VV) acquired by TerraSAR-X data from July 2013 to January 2014 over Tehran plain, Iran. The area has been affected by high rate (> 20 $\mathrm{cm} / \mathrm{yr}$.) of surface subsidence due to groundwater overexploitation. The effectiveness of the method is compared for both agricultural and urban regions affected by land subsidence. Furthermore single pole and optimized polarization results are compared together and with external observations from GPS measurements. The results reveal that using optimum scattering mechanism decreases the ADI values in urban and non-urban regions and increase the PS Candidate pixels (PSC) about three times and subsequently improves the PS density about 50\% more than using single channel datasets.

\section{INTRODUCTION}

Differential Interferometric SAR (DInSAR) technique is a powerful technique to map surface deformations in a wide area with high spatial resolution (Massonnet et al., 1993; Amelung et al., 1999; Lu et al., 2005; Li et al., 2008; Motagh et al., 2010). Because of spatiotemporal decorrelations in the interferograms, it is not possible to always use all pixels from the study area during InSAR processing (Zebker and Villasenor, 1992). To analyse the surface deformation in time and space, time-series analysis of Synthetic Aperture Radar, i.e., Small Baseline Subsets (SBAS) and Persistent Scatterer Interferometric SAR (PSInSAR) is needed to be applied. These methods extend the capabilities of InSAR technique by identifying and processing pixels with negligible effect of decorrelations (Ferretti et al., 2001; Mora et al., 2002; Hooper et al., 2007).

With the recent development in SAR sensors such as ALOS, RadarSat-2, TerraSAR-X, now it's possible to acquire long time-series of SAR Data in multiple polarizations. Studies have shown that using multi-polarization data in PSInSAR method instead of single-polarized data improves the capability of the technique and leads to detection of more PS pixels in deformation maps (Navarro-Sanchez et al., 2010).

In this paper, we proposed a method to apply the polarimetric optimization approach for the Stanford Method for Persistent Scatterers (StaMPS) analysis presented in (Hooper et al., 2007), with the aim to improve its performance in detecting both PSC and PS pixels. The improvement is based on minimizing ADI criteria for selecting PSC pixels in dual polarimetry X-band SAR data, which is followed by reproducing the SAR scenes in optimized scattering mechanism before applying StaMPS method for PS analysis. We test our method for a dataset of 17 dual polarization X-band SAR data (HH/VV) acquired by TerraSAR-X satellite between July 2013 and January 2014 over Tehran plain.

\section{METHODOLOGY}

A pixel is defined as PS, if the phase of the pixel is dominated by a stable scatterer. Ferretti et al., 2001 presented an index called Amplitude Dispersion Index $\left(D_{A}\right)$ that can be employed as an estimation for the phase stability in scatterers with high values of SNR. In this method a low value of ADI, e.g. 0.4, is selected for the threshold and pixels with ADI value less than the threshold are candidate for PS. Hooper et al., 2007, introduced another alternative algorithm which first chooses a higher value of threshold for ADI to select candidates for PS pixels, namely PSC, and then applies a new additional parameter called temporal coherency $\left(\gamma_{x}\right)$ for the final PS selection. In temporal coherency PS pixels are defined as those in which the contribution of the decorrelated parts of the phase including orbital inaccuracies, spatially-correlated and spatially-uncorrelated errors in time is not large to obscure the phase signal (Hooper, 2008). Temporal coherence, which is similar to coherence magnitude, can be estimated as

$$
\gamma_{x}=\frac{1}{N}\left|\sum_{i=1}^{N} \exp \left\{j\left(\psi_{x, i}-\psi_{x, i}-\Delta \psi^{u}{ }_{\theta, x, i}\right)\right\}\right|
$$

Where $\psi_{x, i}$ is the wrapped phase of pixel $\mathrm{x}$ in the $i$ th interferograms, $\psi_{x, i}$ is the estimate for the spatially-correlated terms, $\Delta \psi_{\theta, x, i}^{u}$ is the estimate of the spatially-uncorrelated look angle error term and $\mathrm{N}$ is the number of interferograms. In the following, we extend the ADI for dual-pol data, optimize it to increase the density of PSC pixels and then apply phase stability using equation (1) to detect the final sets of PS pixels. Using Polarimetric Interferometric SAR (PolInSAR) we can produce a $2 \times 2$ scattering matrix $[S]$ for each pixel, which can be vectorized using the Pauli basis (Cloude and Papathanassiou, 1998).

$$
\begin{aligned}
& S=\left[\begin{array}{ll}
S_{h h} & S_{h v} \\
S_{v h} & S_{v v}
\end{array}\right] . \\
& \underline{K}=\frac{1}{\sqrt{2}}\left[S_{h h}+S_{v v}, S_{h h}-S_{v v}\right]^{T}
\end{aligned}
$$

Where $S_{v v}, S_{h h}$ are copular channel, $S_{h v}$ is the cross polar channel and $\mathrm{T}$ is the transpose operator.

Interferogram generation is performed by projecting target vector to a complex vector, $\omega$. Applying the projection vector, which is corresponding to scattering mechanism, results in a scattering coefficient, $\mu$ which defined as:

$$
\mu_{i}=\omega_{i}^{* T} \underline{K}_{i}
$$

Where $*$ represents the conjugate operator.

Now the problem is to find the best projection vector, $\omega$, for each pixel in a SAR image to get better results than single-polar SAR datasets. In (Cloude and Papathanassiou, 1997) the general form of $\omega$ for full-polar SAR data is presented to solve the problem but in order to deal with dual-polarization data, we 
use a reduced projection vector presented in (Navarro-Sanchez et al., 2010):

$$
\underline{\omega}=\left[\cos (\alpha), \sin (\alpha) e^{j \psi}\right]^{T},\left\{\begin{array}{l}
0 \leq \alpha \leq \pi / 2 \\
-\pi \leq \psi \leq \pi
\end{array}\right.
$$

With respect to equation (5), the polarimetric optimization problem of finding the best scattering mechanism is reduced to finding $\alpha$ and $\psi$, two real numbers in a finite range.

Heuristic and meta-heuristic search methods have proved to be powerful in global optimization problems (Xin-She Yang, 2010). In this study we applied Simulated Annealing (SA) to find the optimum polarization mechanism. SA tries to find the values of $\alpha$ and $\psi$, which correspond to the lowest possible value of ADI.

To calculate ADI in polarimetric data using MB-ESM, equation (6), it is sufficient to replace $s$ by polarimetric scattering coefficient $\mu$ as define in Equation (4).

$$
D_{A \text { Pol }}{ }^{2}=N \frac{\sum_{i=1}^{N}\left|\omega^{{ }^{*} T} \underline{K}_{i}\right|^{2}}{\left(\sum_{i=1}^{N}\left|\omega^{{ }^{*} T} \underline{K}_{i}\right|\right)^{2}}
$$

Now the main subject is to find the projection vector providing the optimum value of $D_{A}$.

\section{CASE STUDY}

Tehran, the capital city of Iran, is affected by high rate of land subsidence due to over extraction of ground water resources. Previous studies using InSAR methods showed that the subsidence in Tehran mainly occurs in western part of the Tehran Plain, locally exceeding $20 \mathrm{~cm} /$ year (Motagh et al., 2008). Tehran includes both agricultural and urban regions which helps us assess the performance of the technique for different land uses.

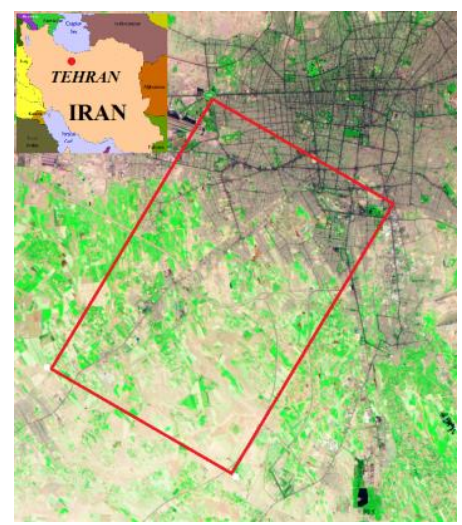

Figure 1. Landsat image of the study area in Tehran, Iran. The red rectangle shows the outline of the cropped TSX images used in this study. The inset in the upper left shows the location of Tehran in Iran.

\section{RESULTS}

By applying SA optimization method we expect that the histogram of ADI values in optimum channel is skewed to lower values. Figure 2 shows a comparison of the histograms of $\mathrm{ADI}$ for $\mathrm{HH}, \mathrm{VV}$ and optimum channels. In this study, pixels with ADI value of lower than 0.4 are selected as PSC. As shown in Figure 2 the use of optimum channel results in greater number of pixels passing the ADI criteria. Since the ADI value of almost every pixel is reduced to its minimum value the chance of pixels to be selected as a PS will increase.

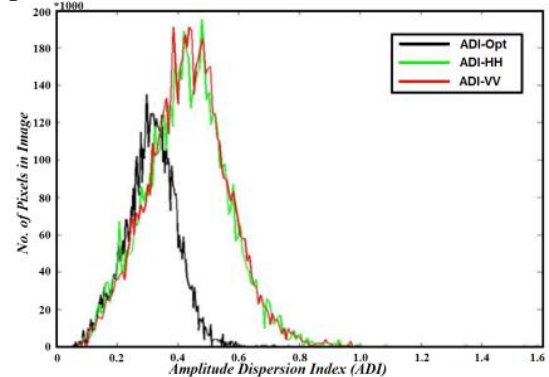

Figure 2. Histograms of ADI for $\mathrm{HH}, \mathrm{VV}$ and optimum channel

After the meaningful increase in density of PSCs due to ADI optimization the next step is to iteratively finalize PS pixels among PSCs using Equation (1).

Number of detected PSC and PS pixels are compared to conventional method using $\mathrm{HH}$ and $\mathrm{VV}$ channels. Table 1 describes the percentage of extra pixels detected by using polarimetric data. Compared to $\mathrm{HH}$ channel PSC density in optimum scattering mechanism has increased about 1.7 times in urban regions and about 2.2 times in non-urban regions. The final PS pixels have increased by about $50 \%$ in both regions. Compared to VV channel our method improved the number of PSC pixels by about three times; the increment in the number of final PS pixels is almost similar to the increment obtained as

\begin{tabular}{|c|c|c|c|c|}
\hline Region Type & $\operatorname{PSC}(\mathbf{H H})$ & PSC (VV) & PS(HH) & PS(VV) \\
\hline Urban & $169 \%$ & $214 \%$ & $53 \%$ & $65 \%$ \\
\hline Non-Urban & $224 \%$ & $215 \%$ & $43 \%$ & $48 \%$ \\
\hline
\end{tabular}
compared to $\mathrm{HH}$ channel, i.e. about $50 \%$.

Table 1. Extra PSC/PS pixels detected by using polarimetric data, compared by HH and VV channel

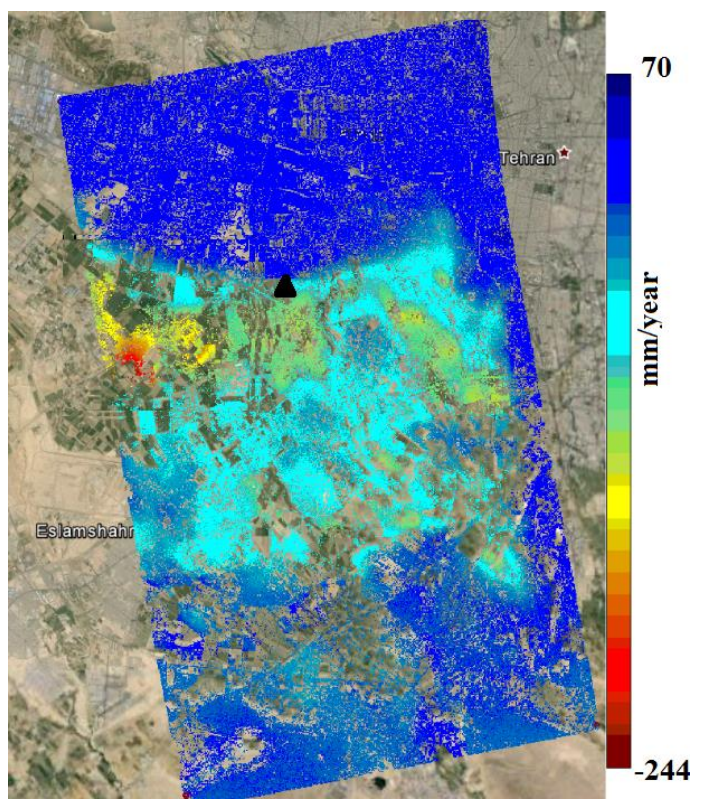

Figure 3. Mean velocity of subsidence obtained by using proposed approach in this paper. The black triangle shows the location of permanent GPS station.

By applying time-series analysis using PSInSAR we can obtain the mean rate of subsidence. Figure 3 presents the mean rate of subsidence obtained by using optimum polarimetric channel 
overlaid on Google Earth image. We can see places in Figure 3 with rates of more than $20 \mathrm{~cm} /$ year which is consistent with previous studies (Motagh et al., 2008).

External observation of a GPS station which is located at southwest of city (see Figure 3) is compared with results of $\mathrm{HH}$, VV and optimum channel. Mean Absolute Error (MAE), Root Mean Square Error (RMSE) and Standard Deviation (Std) are calculated for the difference between various results and GPS observations. Table 2 describes the goodness of the subsidence estimation using different channels used for PS analysis. PsInSAR using both conventional and optimum channel estimate the deformation with accuracy better than $1 \mathrm{~cm}$ as compared to GPS observations, although, using optimum channel shows slightly better MAE, RMSE and Std. than HH and VV results.

Table 2. Numerical comparison between HH/VV and optimum channel with GPS observations

\begin{tabular}{|c|c|c|c|}
\hline Channel & MAE(cm) & Std. (cm) & RMSE (cm) \\
\hline HH & 0.67 & 0.95 & 0.87 \\
\hline VV & 0.71 & 1.01 & 0.96 \\
\hline Optimum & 0.54 & 0.69 & 0.49 \\
\hline
\end{tabular}

\section{CONCLUSION}

In this paper, we evaluated a method to combine Amplitude Dispersion Index optimization with phase stability criteria and temporal coherence to improve the performance of StaMPS method for PSInSAR analysis using dual polarimtery SAR data. Minimizing the ADI values in polarimetric data increases the number of PSCs significantly. Our finding shows that implementing phase stability criteria after this step meaningfully improves the density of PSC/PS pixels in both urban and non-urban regions. In particular in areas with odd bouncing scattering (e.g. farmland regions) our findings shows that the use of polarimetry data increases the chance of finding more PS pixels for deformation analysis. The future researches should focus on implementing the method proposed here for full-polarimetry data in order to better estimate dominant scattering mechanism at each pixel which helps improve spatial sampling of deformation signal associated with different land cover. Moreover, we can also implement the polarimetric optimization for slowly decorrelating filtered phase (SDFP) pixels used in the SBAS technique (Hooper, 2008) to further improve its performance for different models of ground scattering.

\section{ACKNOWLEDGMENTS}

Copyright of TerraSAR-X data belongs to German Aerospace Agency (DLR) and were provided by the project Motagh_XTI_LAND505.

\section{REFERENCES}

Amelung, F., Galloway, D.L., Bell, J.W., Zebker, H.A., Laczniak, R.J., 1999. Sensing the ups and downs of Las Vegas: InSAR reveals structural control of land subsidence and aquifer-system deformation. Geology 27, 483-486.

Cloude, S.R., Papathanassiou, K.P., 1998. Polarimetric SAR interferometry. Geosci. Remote Sens. IEEE Trans. On 36, 1551-1565.

Cloude, S.R., Papathanassiou, K.P., 1997. Polarimetric optimisation in radar interferometry. Electron. Lett. 33, 1176-1178.
Ferretti, A., Prati, C., Rocca, F., 2001. Permanent scatterers in SAR interferometry. Geosci. Remote Sens. IEEE Trans. On 39, 8-20.

Hooper, A., 2008. A multi-temporal InSAR method incorporating both persistent scatterer and small baseline approaches. Geophys. Res. Lett. 35 . doi:10.1029/2008GL034654

Hooper, A., Segall, P., Zebker, H., 2007. Persistent scatterer interferometric synthetic aperture radar for crustal deformation analysis, with application to Volcán Alcedo, Galápagos. J. Geophys. Res. 112. doi:10.1029/2006JB004763

Li, Z., Feng, W., Xu, Z., Cross, P., Zhang, J., 2008. The 1998 Mw 5.7 Zhangbei-Shangyi (China) earthquake revisited: A buried thrust fault revealed with interferometric synthetic aperture radar. Geochem. Geophys. Geosystems 9.

Lu, Z., Masterlark, T., Dzurisin, D., 2005. Interferometric synthetic aperture radar study of Okmok volcano, Alaska, 1992-2003: Magma supply dynamics and postemplacement lava flow deformation. J. Geophys. Res. Solid Earth 1978-2012 110.

Massonnet, D., Rossi, M., Carmona, C., Adragna, F., Peltzer, G., Feigl, K., Rabaute, T., 1993. The displacement field of the Landers earthquake mapped by radar interferometry. Nature 364, 138-142. doi:10.1038/364138a0

Mora, O., Lanari, R., Mallorquí, J.J., Berardino, P., Sansosti, E., 2002. A new algorithm for monitoring localized deformation phenomena based on small baseline differential SAR interferograms, in: Geoscience and Remote Sensing Symposium, 2002. IGARSS '02. 2002 IEEE International. pp. 1237-1239 vol.2. doi:10.1109/IGARSS.2002.1025900

Motagh, M., Schurr, B., Anderssohn, J., Cailleau, B., Walter, T.R., Wang, R., Villotte, J.-P., 2010. Subduction earthquake deformation associated with 14 November 2007, Mw 7.8 Tocopilla earthquake in Chile: Results from InSAR and aftershocks. Tectonophysics 490, $60-$ 68. doi:http://dx.doi.org/10.1016/j.tecto.2010.04.033

Motagh, M., Walter, T.R., Sharifi, M.A., Fielding, E., Schenk, A., Anderssohn, J., Zschau, J., 2008. Land ubsidence in Iran caused by widespread water reservoir overexploitation. Geophys. Res. Lett.

Navarro-Sanchez, V.D., Lopez-Sanchez, J.M., VicenteGuijalba, F., 2010. A Contribution of Polarimetry to Satellite Differential SAR Interferometry: Increasing the Number of Pixel Candidates. IEEE Geosci. Remote Sens. Lett. 7, 276-280. doi:10.1109/LGRS.2009.2033013

Neumann, M., Ferro-Famil, L., Reigber, A., 2008. Multibaseline polarimetric SAR interferometry coherence optimization. Geosci. Remote Sens. Lett. IEEE 5, 93-97.

Xin-She Yang, 2010. Engineering Optimization: An Introduction with Metaheuristic Applications. John Wiley \& Sons.

Zebker, H.A., Villasenor, J., 1992. Decorrelation in interferometric radar echoes. Geosci. Remote Sens. IEEE Trans. On 30, 950-959. doi:10.1109/36.175330 